\title{
Web-GIS Based Visualization System of Predicted Ground Vibration Induced by Blasting in Urban Quarry Sites
}

\author{
Youhei Kawamura' ${ }^{1}$, Yoshio Moriyama ${ }^{1}$, Hyongdoo Jang ${ }^{2}$ \\ ${ }^{1}$ Department of Earth Resource Engineering and Environmental Science, Akita University, Akita, Japan \\ ${ }^{2}$ Minerals, Energy and Chemical Engineering, WASM, Curtin University, Kalgoorlie, Australia \\ Email: y.kawamura@gipc.akita-u.ac.jp
}

How to cite this paper: Kawamura, Y., Moriyama, Y. and Jang, H. (2019) Web-GIS Based Visualization System of Predicted Ground Vibration Induced by Blasting in Urban Quarry Sites. Journal of Geographic Information System, 11, 17-31.

https://doi.org/10.4236/igis.2019.111002

Received: December 27, 2018

Accepted: January 29, 2019

Published: February 1, 2019

Copyright $\odot 2019$ by author(s) and Scientific Research Publishing Inc. This work is licensed under the Creative Commons Attribution International License (CC BY 4.0).

http://creativecommons.org/licenses/by/4.0/

\begin{abstract}
Blasting is routinely carried out at various resource extraction sites, even in urban areas. As a consequence of this, residents around urban quarry sites are affected by ground vibration induced by blasting on a regular basis. In this study, a prediction and visualization system for ground vibrations is developed for the purpose of reducing the adverse psychological effects of blasting. The system consists of predicting ground vibration using an Artificial Neural Network (ANN) and visualizing it on an online map using Web-GIS. A prediction model using ANN that learned the optimum weight by taking 50 sets of data indicated a regression value of 0.859 and a Mean Square Error (MSE) of 0.0228. Compared with previous researches, these values are not bad results. Peak Particle Velocity (PPV) was used as a metric to measure ground vibration intensity. A color contour is generated using GIS tools based on the PPV value of each prediction point. The system is completed by overlaying the contour onto a basic map in a website. The basic map shows the surrounding area through the use of Google Maps data. This system can be used by anyone with access to the internet and a browser, requiring no special software or hardware. In addition, mining operations can utilize the data to modify blasting design and planning to minimize ground vibration. In conclusion, this system has the potential to alleviate the worries of surrounding residents caused by ground vibrations from blasting due to the fact that they can personally check the predicted vibration around their locale. Furthermore, since this data will be publicly available on the internet, it is also possible that this system can contribute to research in other fields.
\end{abstract}

\section{Keywords}

ANN, Prediction Model, PPV, Web-GIS, Ground Vibration Visualization 


\section{Introduction}

Blasting is one of the excavating technique and the operation that breaks rocks using explosive. Blasting is routinely carried out at various resource extraction sites. As a consequence, when an instantaneous blast is caused by using large amounts of explosives, ground vibrations will propagate from the site to the adjacent areas. Besides, blasting generates problems such as fly rock, dust and fumes. The company which is running the site needs to exercise caution and apply solutions so that these problems do not adversely affect the surrounding environment. Specifically, in this study area, there are residential areas around a quarry site, so they must pay exceptionally more attention to these problems. Even if residential houses and other structures are not significantly affected, the ground vibration induced by blasting can induce anxiety and/or discomfort to residents. One of the main reasons for this effect is that there is only little information that nearby residents know about currently ongoing blasting operations. In previous researches on residents' response to ground vibration induced by blasting, it has been observed that mental stress such as anxiety of the surrounding residents can be alleviated by providing blasting information prior to blasting activity [1]. In previous researches on ground vibration induced by blasting, these have been done that are aimed to develop a ground vibration prediction model by using nonlinear expressions and artificial intelligence [2] [3] [4] [5]. However, few researches have been conducted for the purpose of developing a system that can reduce the influences on neighboring residences using prediction of ground vibration that is induced by blasting [6] [7].

In this research, we constructed a system that visualizes ground vibration on a map, using the Mikurahana quarry site located in the town of Hachirogata in Akita Prefecture, Japan as the experiment site. At first, as a construction procedure of this system, prediction model of ground vibration is generated by using Artificial Neural Network (ANN) $)^{1}$ that is one of the artificial intelligence. Then, it will be possible to predict at multiple points around the quarry site using generated prediction model. A contour that represents ground vibration intensity is drawn by using Geographic Information System (GIS) that integrates geospatial information. Finally, the contour is visualized on the map in the web area using Web-GIS. With this, any person who has access to a web browser can see the map. GIS and Web-GIS are already being used for land use maps, geology and hazard maps, and are used for disaster simulation and research on dynamic management system of resource extraction sites [8] [9] [10] [11] [12]. The proposed system design is shown in Figure 1. By visualizing the predicted vibration intensity of the ground vibration that is to be induced by blasting on the map found in an online website, anyone can access the information with a browser, and the predicted vibration intensity of the ground vibration at each point in the vicinity of the upcoming blast can be known. Using this system, there is a possibility of reducing the mental stress experienced by the surrounding residents.

${ }^{1}$ Application of artificial intelligence that mathematically simulates the logical processes of the human brain. 


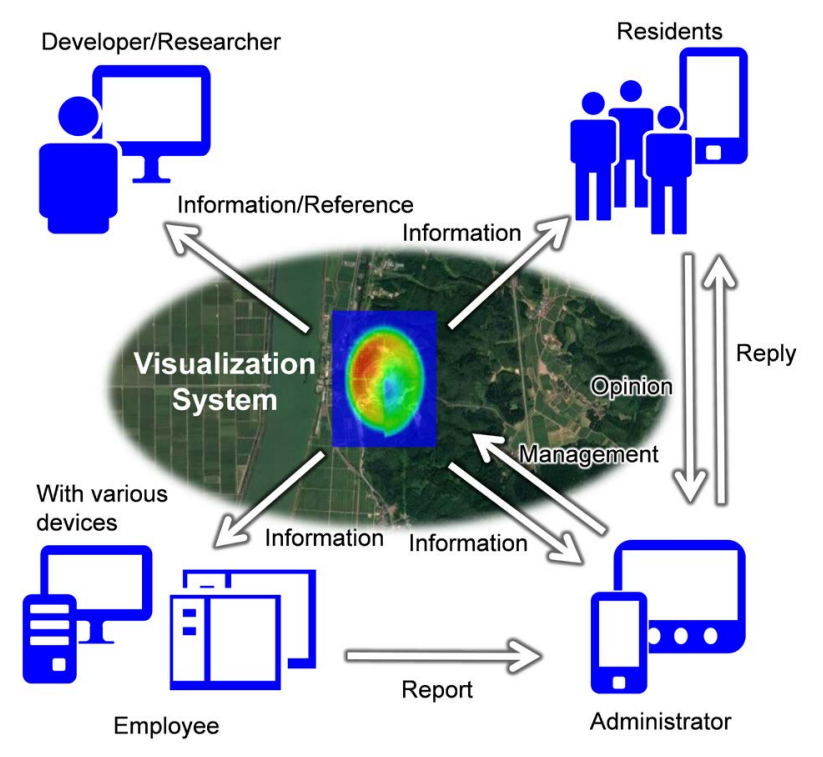

Figure 1. System design

Also, since the system is relatively eased to implement and use, it can be utilized in various fields such as operations management and further research.

\section{System Overview}

The implementation procedure of the system is shown in Figure 2. It is possible that construction procedure is intuitively understanding because of Figure 2. The detailed data handling is shown in Figure 3. Data handling consists of "PPV prediction by ANN" part and "Visualization" part. Further, "PPV prediction by ANN" is divided into "On site experiment for data accumulation", "Learning phase" and "Calculate PPV for each case". In the data accumulation, the Ground vibration data is first collected by on-site accelerometers. The ground vibration data obtained at the voltage value $(\mathrm{V}$ or $\mathrm{mV})$ and finally converted into the velocity. Peak Particle Velocity (PPV) ${ }^{2}$ is extracted from velocity data [13]. The collected data is fed to the ANN which is one of the machine learning method, and a prediction model of ground vibration induced by blasting is generated in the learning phase. Prediction of PPV in specific conditions (latitude/longitude, blast design, geographical condition etc.) is estimated using the prediction model in the PPV calculation. Also, "Visualization" part is divided into "GIS" and "Web-GIS". In the GIS part, utilizing GIS and its ability to handle geospatial information and overlay it along with other various information on the map, it is possible to visualize the predicted PPV linked to the latitude and longitude on a digital map. Specifically, ground vibration is expressed as color contours based on the PPV value of each predicted PPV. In order to use map created by GIS, a dedicated application is required, so it can be used only within a limited range in the Web-GIS part. Therefore, in order to expand the range, the map is generated and hosted online using Web-GIS. In the subsequent chapters, the measurement ${ }^{2}$ Peak value of particle velocity. It is used as a general index for evaluating ground vibration intensity in this field. 


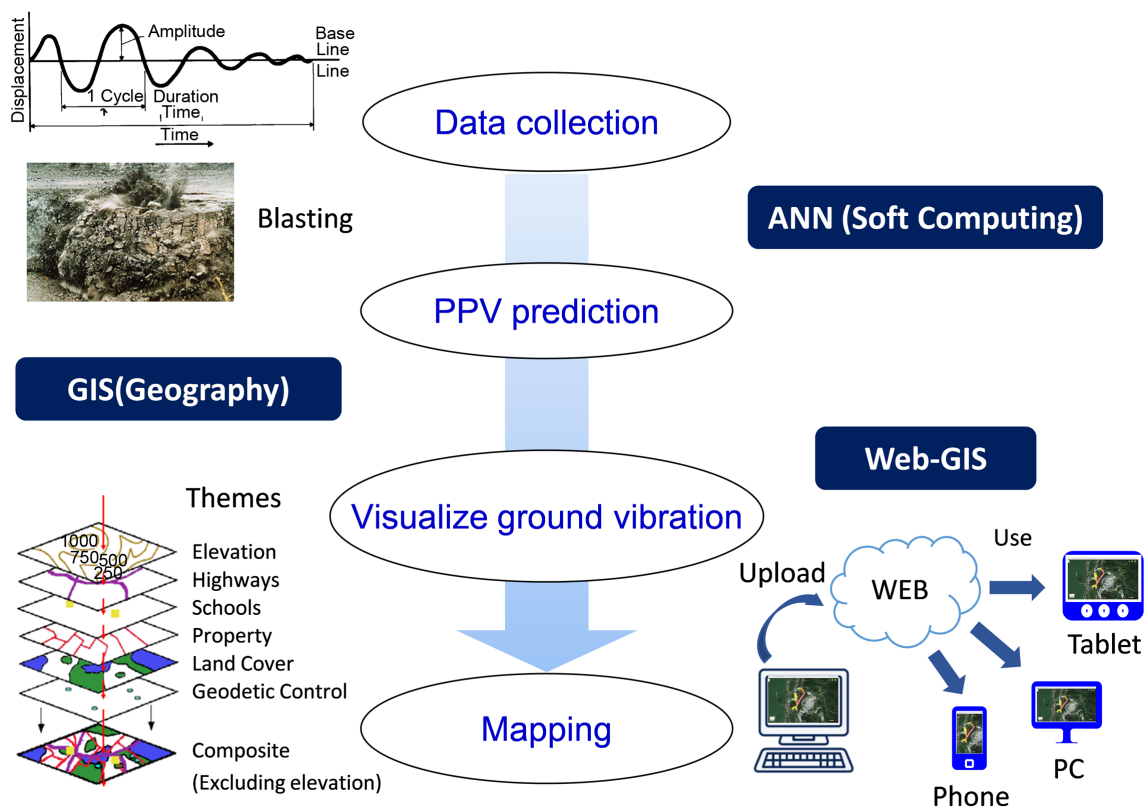

Figure 2. System implementation procedure.

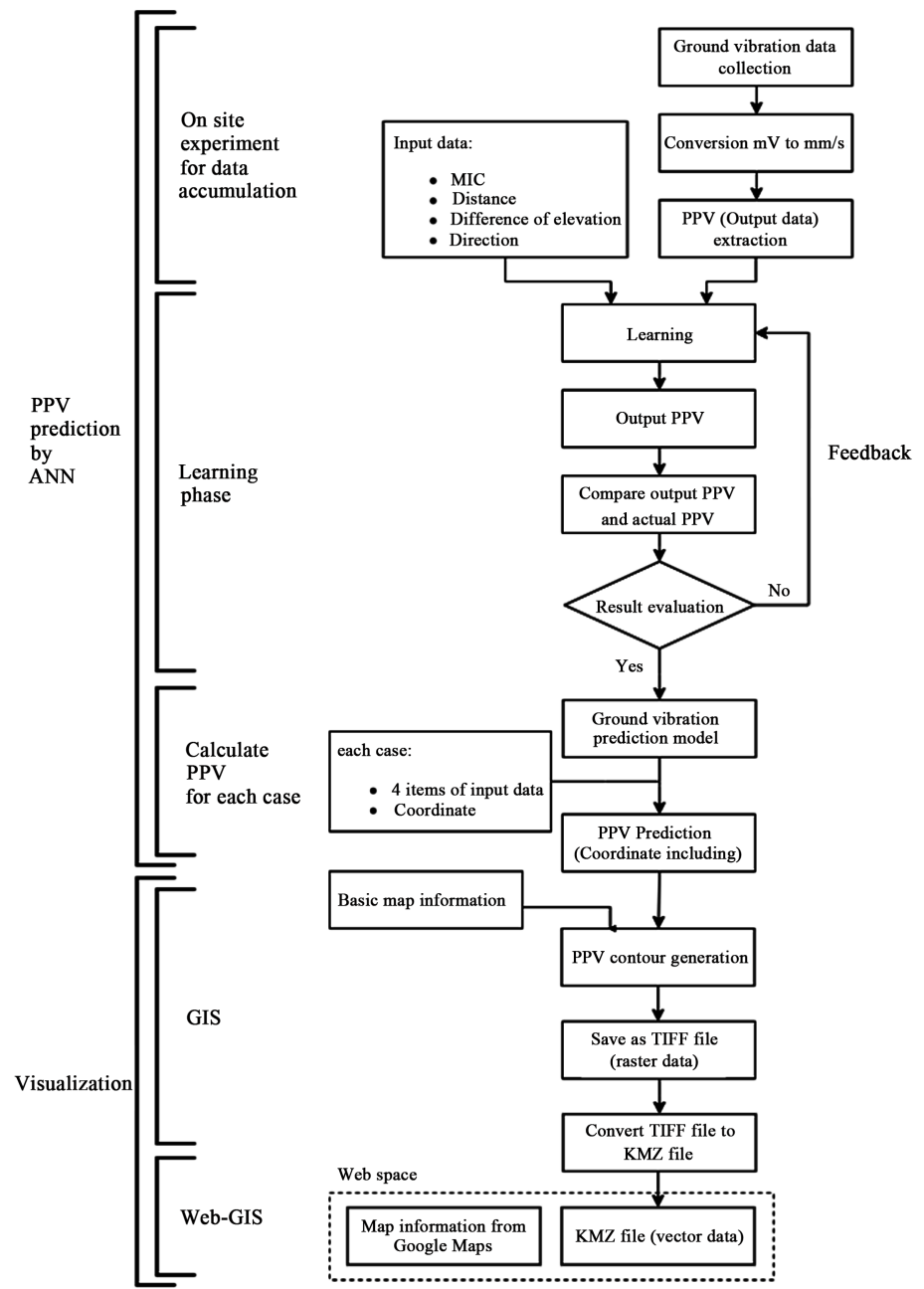

Figure 3. Data processing in this study. 
of ground vibration, the generation of prediction model using ANN, and the visualization system by Web-GIS will be explained in detail.

\section{Measurement of Ground Vibration and Generation of Prediction Model Using ANN}

In the vicinity of the Mikurahana quarry site, there are residences located 10 - 20 meters from the quarry site. Under this circumstance, the study has been conducted at the Mikurahana quarry site as a feasible experiment site. Schematic diagram of apparatus to measure ground vibration is shown in Figure 4. Two types of sensors were used, a tri-axial micro vibration detector and a mono-axial accelerometer, collecting the ground vibration data. The points where measurements were made around the quarry site are indicated by yellow markers in Figure 5. Ground vibration measurement is acquired as a voltage value (V or $\mathrm{mV}$ ), and is multiplied by a calibration coefficient inherent to the tri-axial micro vibration detector to convert this voltage value to acceleration $\left(\mathrm{mm} / \mathrm{s}^{2}\right)$. The mono-axial accelerometers with a different calibration coefficient are adjusted to the same coefficient as the tri-axial detector by using an amplifier. The ground vibration data is finally converted into velocity and PPV by taking integration, and is then used as a general index for evaluating ground vibration intensity. Since velocity is not affected by changes in geological conditions unlike acceleration and displacement, consistency and predictability are can be maintained even if conditions such as rock type and distance change [14]. Figure 6 shows an example of ground vibration data measured at the Mikurahana quarry site in terms of voltage values. The vertical axis of the graph represents the voltage value, and the horizontal axis represents the measurement time, respectively. One measurement duration was set to 1.6 seconds. The sampling frequency was 10 $\mathrm{kHz}$. A speed-time graph was prepared using a spreadsheet software and PPV was interpolated from the speed-time graph. Using Maximum Instantaneous Charge (MIC) $)^{3}$, distance, direction, elevation difference values as input data and

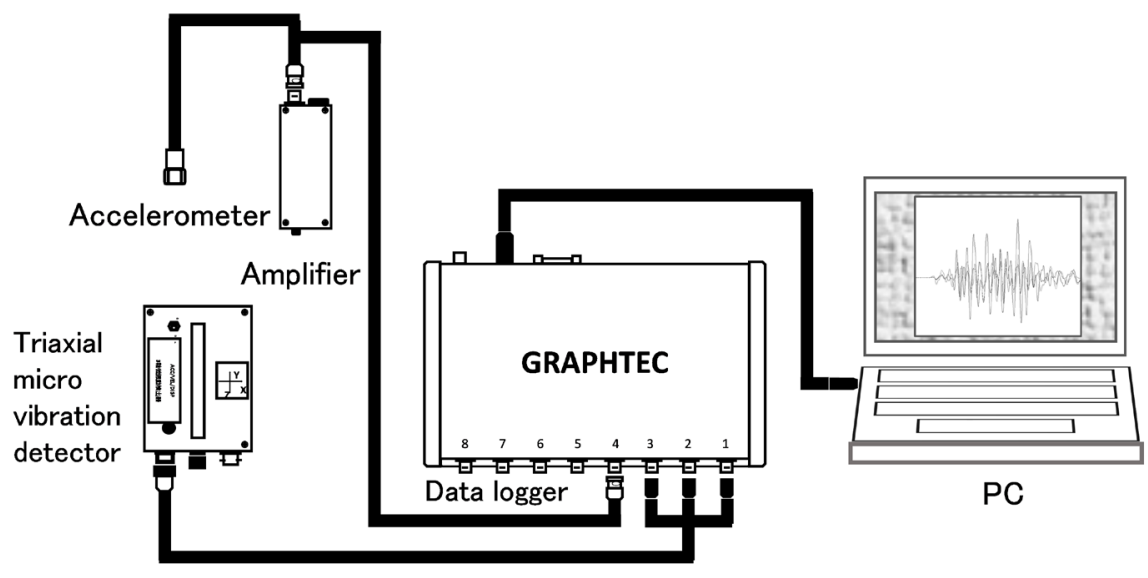

Figure 4. Schematic diagram of ground vibration measurement apparatus.

${ }^{3}$ Amount of explosive that detonating simultaneously within 8 milliseconds. 


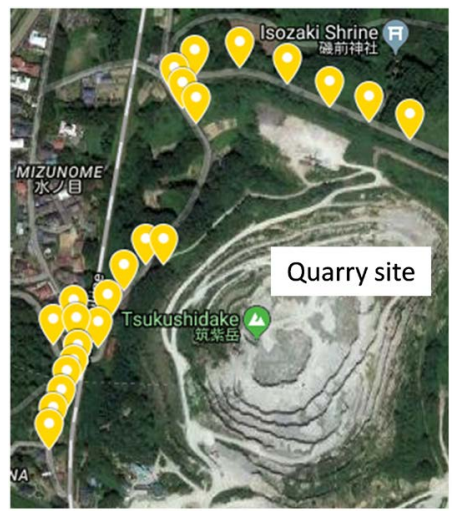

Figure 5. Points of ground vibration measurement.

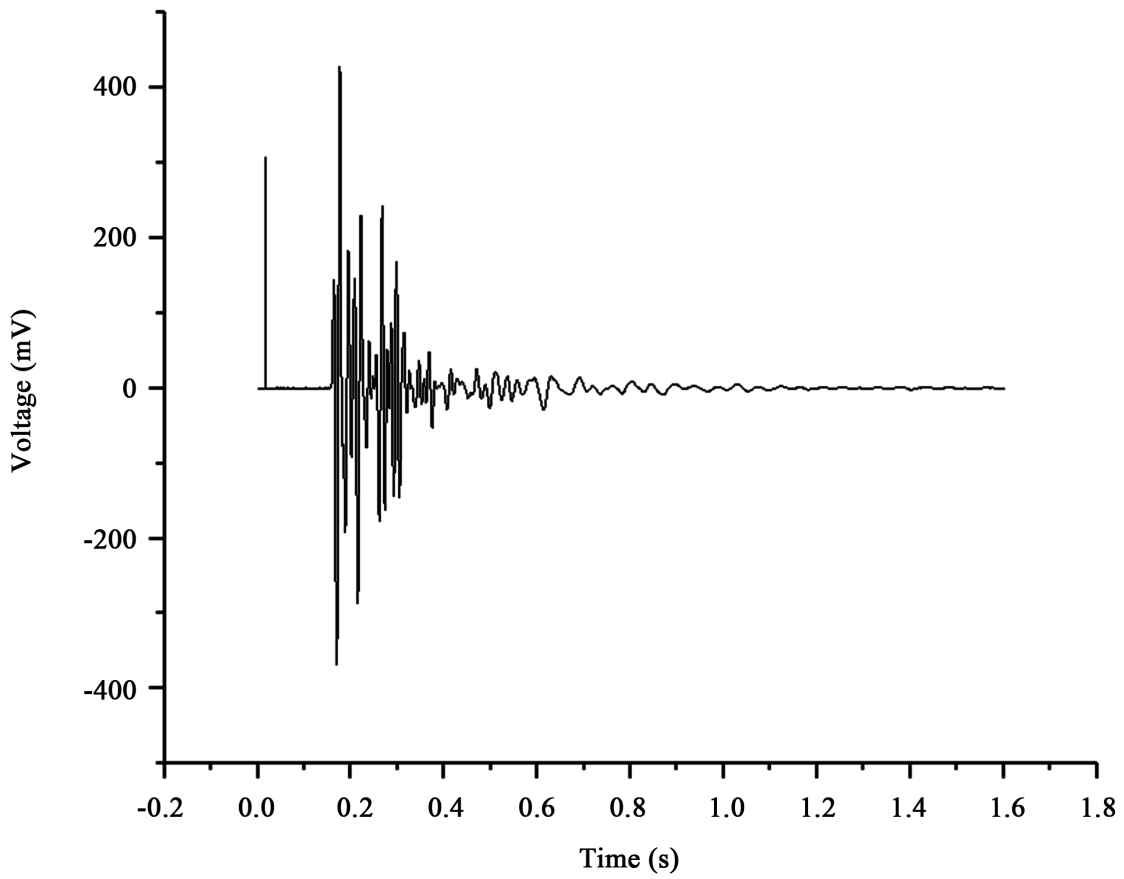

Figure 6. Example of measured ground vibration data.

measured PPV as output data (target), a prediction model of ground vibration induced by blasting using ANN was generated using MATLAB. Idea of MIC is amount of explosive that detonating simultaneously within 8 milliseconds. The ANN is an application of artificial intelligence that mathematically simulates the logical processes of the human brain. In particular, the concept of ANN belongs in machine learning, and by accumulating and learning from data as "knowledge", it is a system that can perform certain simulations such as classification and regression. Since a prediction model using ANN shows relatively higher performance as compared to standard empirical estimation, ANN was used to generate the prediction model in this research [2] [3] [4] [5]. For example, coefficient of determination $\left(\mathrm{R}^{2}\right)$ was 0.7987 when using nonlinear expressions and 0.9493 when using ANN on the predicted performance comparison in a previous research. Therefore, the advantage of the prediction model using ANN was in- 
dicated. The prediction model using ANN is high prediction performance because it can generate a prediction model specialized for the site. 50-sets of data consisting of input data and output data were prepared as data sets. This data set was separated into learning data, test data and verification data at a ratio of 30:10:10. Figure 7 shows a conceptual diagram of a prediction model of ground vibration using ANN. In this research, a three-layered neural network with a single hidden layer was designed. The learning procedure of the neural network is shown below. In the input layer, each value of the respective data is considered input. Weight is applied when proceeding to the hidden layer, and their sum value is outputted to the hidden layer. The predicted PPV is then sent to the output layer based on the total value of the hidden layer. Comparison between the predicted PPV with the measured value (measured PPV) then takes place. This result is then fed back to the neural network where the system improves prediction accuracy by changing the weight so that the error from comparison becomes progressively smaller. The weight is a parameter that determines the importance of the data. The weight is initially set as an appropriately fitted value. The cycle of data input, prediction, comparison and feedback modifies the weight so as to reduce the error. In other words, it could be said that the neural network is "learning" to optimize the weight.

Equation (1) shows the basic calculation model of the neural network. Values of previous layers are multiplied by coefficients and added together.

$$
l^{(k+1)}=\sigma^{(k+1)}\left(W^{(k+1)} l^{(k)}+b^{(k+1)}\right)
$$

The value attached to each symbol as an exponent denotes the number of the layers. This is a recurring formula for obtaining the value $l^{(k+1)}$ of the $(k+1)$ layer from the value $l^{(k)}$ of the $\mathrm{k}$ layer. Where $W$ is the weight and $b$ is a bias that does not affect the nodes of the previous layer. $\sigma$ is an activation function, and it has the function of adjusting the value $(W l+b)$, before it is sent to the next layer. Weight $W$ of Equation (1) is learned iteratively using learning data to make it the optimum value.

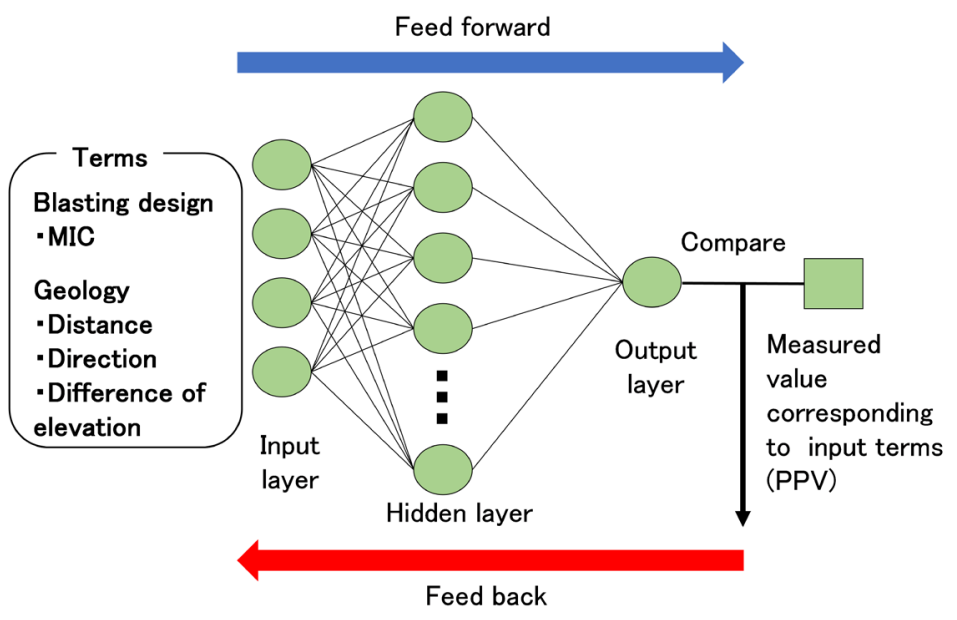

Figure 7. Conceptual diagram of artificial neural network. 
After teaching the neural network using learning data, performance evaluation and generalization of the neural network using test data and verification data is done. Both the test data and the verification data are used to further improve the neural network. The evaluation result is displayed as a numerical value of regression and Mean Square Error (MSE). A value closer to 1 is desired for the regression value, and a value closer to 0 is desirable for the MSE. The number of artificial neurons in the hidden layer $(4,6, \cdots, 12)$ is divided and compared them to determine the optimum number of artificial neurons. Table 1 shows the comparison results of each prediction model. Based on the regression ratio and the MSE, it can be seen that the prediction model with 10 hidden layer artificial neurons showed the best performance. Figure 8 shows a regression analysis graph of a prediction model in which the number of artificial neurons in the hidden layer is 10 . The vertical axis shows the predicted PPV $(\mathrm{mm} / \mathrm{s})$ based on the input, and the horizontal axis shows the measured PPV $(\mathrm{mm} / \mathrm{s})$ corresponding to the input. In this graph, a plot of each data and an approximate line are drawn, and it shows the correlation between the predicted PPV and the measured PPV. The regression rate is higher as the approximate line and the plot overlap. The regression ratio of each data is 0.972 for learning data, 0.860 for verification data, 0.859 for test data and 0.873 for all data sets. These regression rates close to 0.9 , which in itself is desirable, but it is not as high of a regression ratio as compared with previous research. The average value of correlation coefficient cited from five previous researches is 0.981 [5] [13] [15] [16] [17]. The suspected reason for this discrepancy is data shortage. Despite this, it was decided that the data was sufficient for generating a prediction model with10 hidden layer artificial neurons as a proof-of-concept.

Table 1. Comparison result of prediction model.

\begin{tabular}{|c|c|c|c|}
\hline Data Name & Number of Artificial Neuron & MSE & $\mathrm{R}$ Value \\
\hline Learning Data & & 0.0091 & 0.831 \\
\hline Validation Data & 4 & 0.0477 & 0.705 \\
\hline Test Data & & 0.0437 & 0.767 \\
\hline Learning Data & & 0.0121 & 0.820 \\
\hline Validation Data & 6 & 0.0093 & 0.898 \\
\hline Test Data & & 0.0278 & 0.943 \\
\hline Learning Data & & 0.0046 & 0.943 \\
\hline Validation Data & 8 & 0.0259 & 0.844 \\
\hline Test Data & & 0.0242 & 0.818 \\
\hline Learning Data & & 0.0020 & 0.972 \\
\hline Validation Data & 10 & 0.0327 & 0.860 \\
\hline Test Data & & 0.0228 & 0.859 \\
\hline Learning Data & & 0.0042 & 0.951 \\
\hline Validation Data & 12 & 0.0210 & 0.899 \\
\hline Test Data & & 0.0195 & 0.794 \\
\hline
\end{tabular}



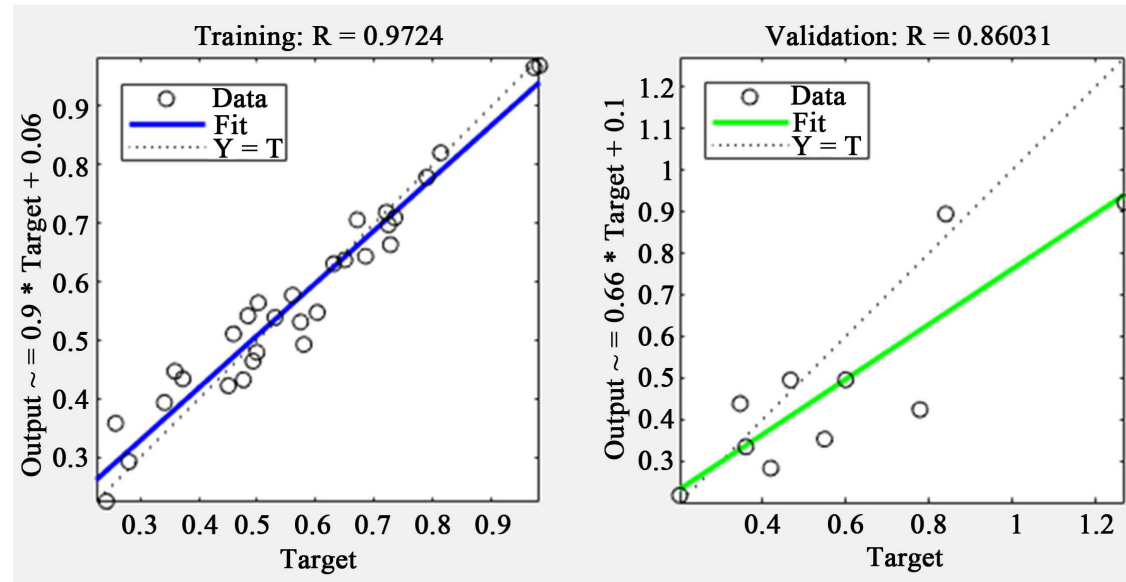

Test: $\mathbf{R}=0.8589$
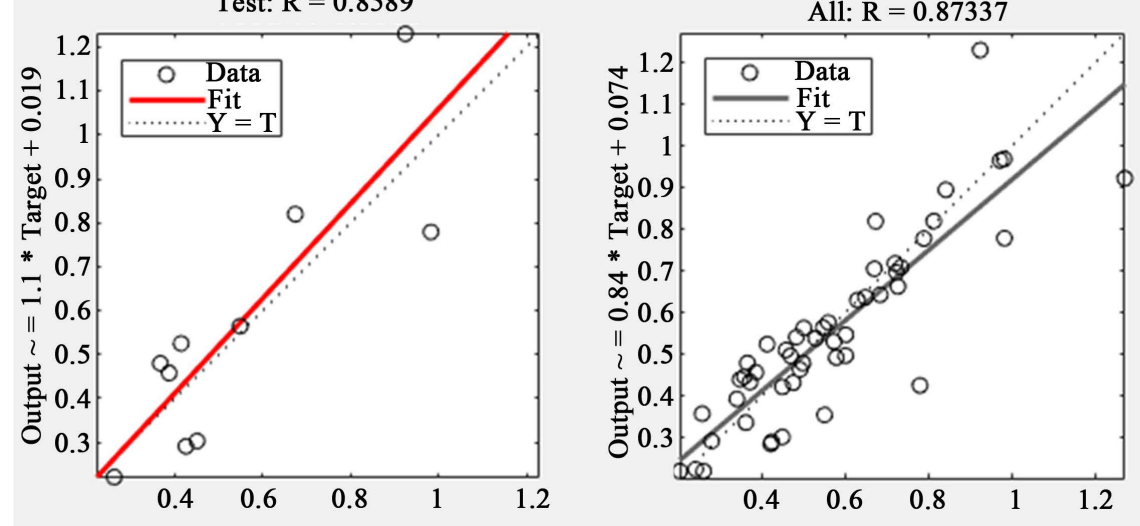

Figure 8. Regression analysis graph.

\section{Predicted Vibration Visualization System Using Web-GIS Technology}

The system overview of generating a predicted vibration visualization system using Web-GIS is shown in Figure 9. Firstly, the PPV is estimated at multiple points around the Mikurahana quarry site using the prediction model of ground vibration. In this experiment, the PPV value in around 10,000 points centered on the lowermost layer of the Mikurahana quarry site were estimated. The standard deviation of the learning data's MIC and the elevation values were small, so a constant is used as per prediction condition. On the other hand, the distance and direction were used values for each point. The prediction result was saved as a CSV file together with the latitude and longitude information of each point. Negative values also exist in the prediction results, due to the lower prediction accuracy in the southeast direction from the center point. A possible reason for this negative value may be that it is a point where the ground vibration due to blasting doesn't reach. Another possible reason is the lack of data in the mountainous areas around the experiment site. It is necessary to increase the data set hereafter. However, the mountain area is not necessary to care in this research because there are no people living in this area. 


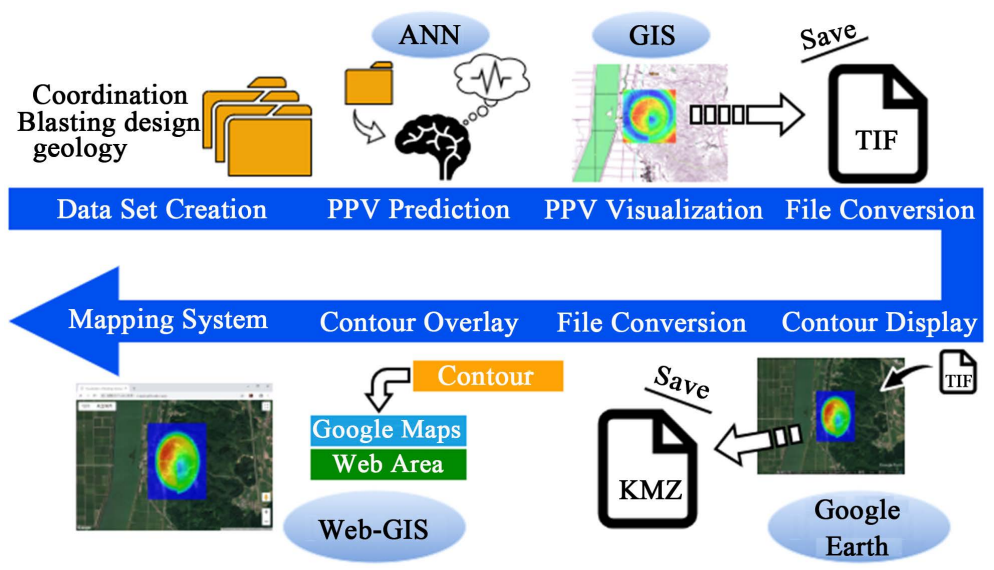

Figure 9. Overview of creation of predictive vibration visualization system using Web-GIS.

Secondly, the predicted PPV values at multiple points around the Mikurahana quarry site are visualized on a map using tool of GIS. Basic map information around the Mikurahana quarry site used in GIS was downloaded from the website of Geographical Survey Institute, Japan. Using the basic map information viewer, basic map information was converted to a shapefile and imported to GIS. The CSV files containing the prediction results for PPV values were also imported. Figure 10 shows the prediction result of PPV values displayed as markers on the basic map. The different marker colors are denoting each of the 5 of the CSV files that were imported. Contours were created based on PPV values at each point using a function that converts marker information, which is vector data, to raster data. Figure 11 shows the PPV contour created. The closer the contour is to blue, the smaller the vibration, and the closer it is to red, the greater the vibration. Since, PPV is proportional to ground vibration intensity, it can be said that the contour also represents the ground vibration intensity. As described above, since the prediction performance of the prediction model is not high, there is an irregularity in the contour color distribution.

In order to solve this problem, the study deems it necessary to increase the amount of data. Since the negative values reflect the magnitude of the numerical value irrespective of the sign, it is treated as 0 , and the correction processing is performed so that the outside range of the minimum vibration (blue) has the same color. Although predicted vibration could be visualized, in this state, a person without access to GIS tools cannot see the visualized result of predicted vibration. Therefore, it is necessary to construct a system that anyone can use by overlaying visualized contours on the web-based map. Prediction vibration of ground vibration is visualized on the web-based map using Web-GIS. The basic map to be used on the website was acquired from Google Maps. Contours generated with GIS needed to be converted to Keyhole Markup Language (KML) file format for ease of use in Hyper Text Markup Language (HTML) language.

${ }^{4}$ One of markup languages and it is used to display geographic data in Google maps and Google Earth. 


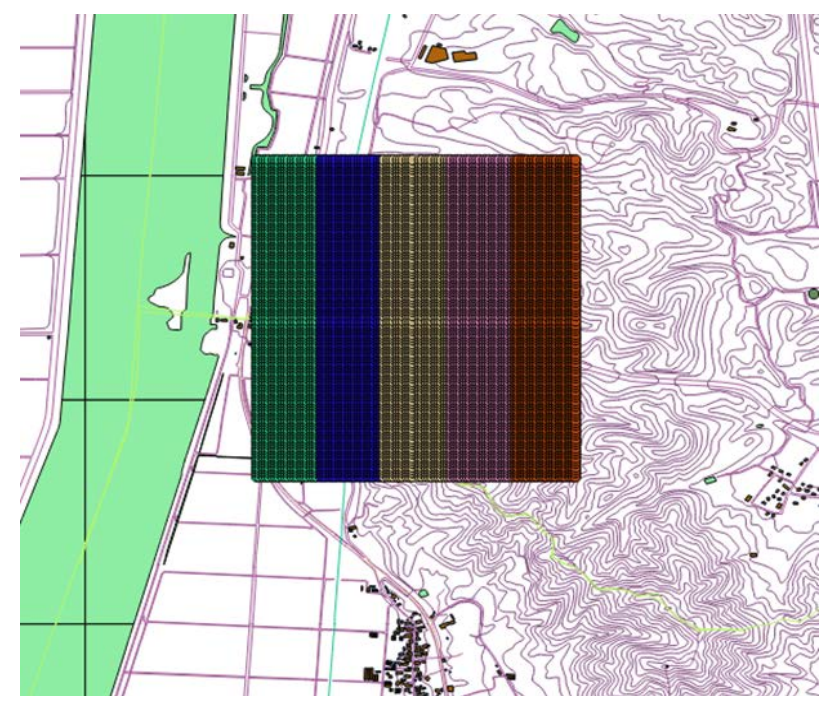

Figure 10. Markers based on predicted PPV.

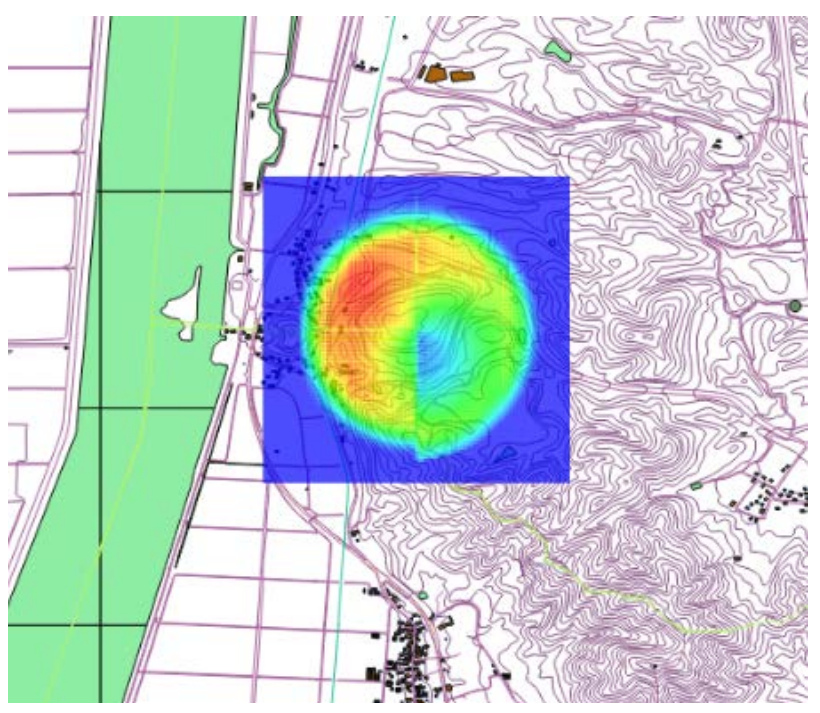

Figure 11. Contour of PPV.

To achieve this problem, contour raster data is converted into Tagged Image File Format (TIFF) and displayed once on Google Earth. Then, the contours displayed in Google Earth are converted to $\mathrm{KMZ}^{5}$ file format. Here, the KMZ file is composed of a main KML file and possibly some support files, which are packaged as a Zip file. A folder named KMZ was created in the same hierarchy as the index.html file for the purpose of editing the Web area, and KMZ file is then saved in that folder. Finally, a program that calls the basic map from the Google map was written in HTML language in the index.html file as well as a program to read the KMZ file from the KMZ folder. As a result, contours can be overlaid on the basic map, and so the ground vibration prediction visualization can now be seen on the website, as shown in Figure 12. This figure is a system example that was visualized a predicted vibration under a particular condition. Also, URL ${ }^{5}$ One of the Zip file format. It is composed of a main KML file and possibly some support files. 


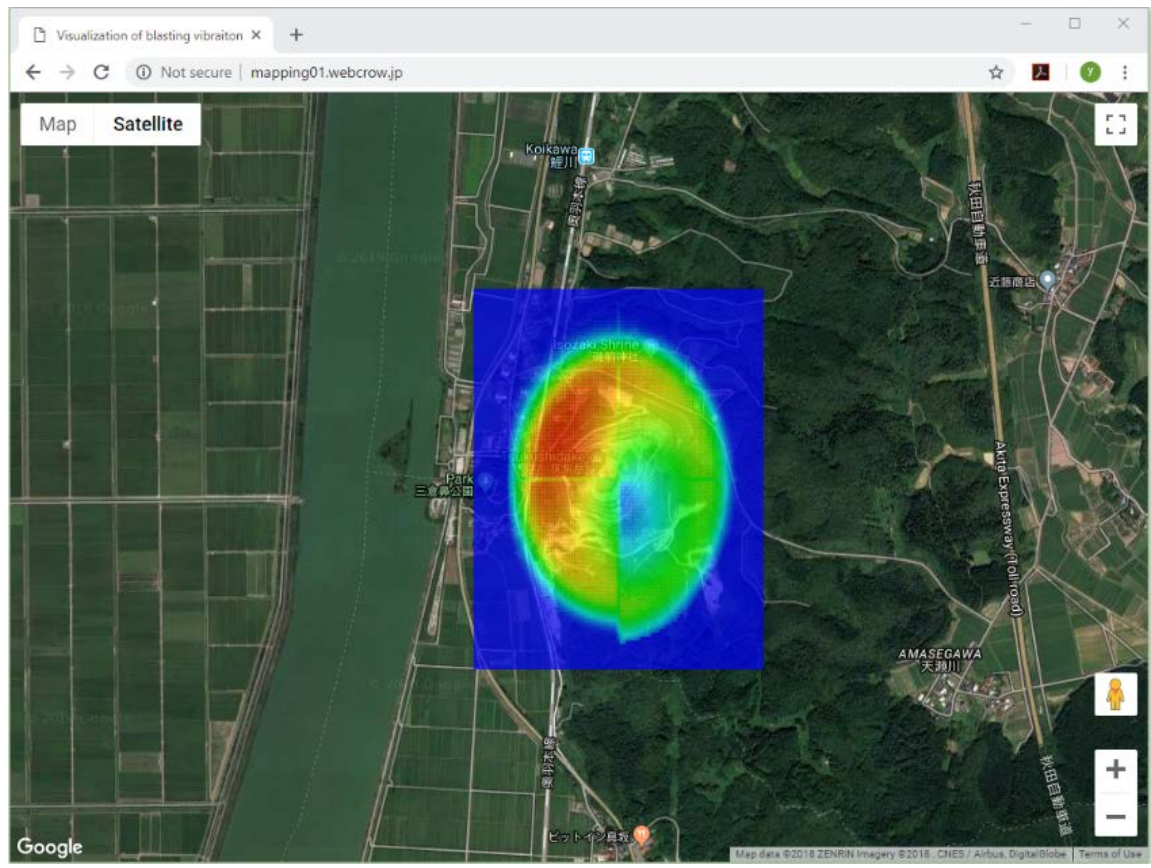

Figure 12. Screen shot of predictive vibration visualization system using Web-GIS (URL: http://mapping01.webcrow.jp/).

of website attached to figure title. Contour shape has been transformed by overlaying contours created with GIS on the website. This is considered to be caused by the fact that the spatial reference system of GIS and Google Maps is different and the coordinates are shifted. Here, it is shown that the difference in vibration level due to the change of blasting conditions taking a residential house around the Mikurahana quarry site as an example. The contour blasting condition of Figure 11 is MIC $24.8 \mathrm{~kg}$, distance $16.6 \mathrm{~m}-830.1 \mathrm{~m}$, difference of elevation 23 $\mathrm{m}$, direction $0^{\circ}-180^{\circ}$. The distance indicates the distance from the blasting point to the predicted point. The direction indicates the deviation of the direction of the predicted point when the perpendicular of the normal plane is taken as 0 degrees when viewed from the blasting point. Only the value of MIC was changed to compare the vibration level transmitted to the residential house. It is possible to change MIC by changing the number of blast holes and the detonation interval of blast holes. The conditions for MIC of $22 \mathrm{~kg}$ and $20 \mathrm{~kg}$ were taken. Screenshots of making contour under each blasting condition and enlarging a specific residential house as a comparison object is shown in Figure 13.

In addition, the legend of the color contour was attached. Ground vibration exceeding $1.4 \mathrm{~mm} / \mathrm{s}$ is transmitted to the target residential house when the MIC is $24.8 \mathrm{~kg}$ (enlarged view on the left). Therefore, MIC is set to $22 \mathrm{~kg}$ or $20 \mathrm{~kg}$ by reducing the amount of explosive directly or by increasing the detonation interval per hole. This shows that the vibration level decreases to green and blue. It is possible to adjust the blast design and optimize the vibration level by using this method. Also, it is possible to announce the vibration level of the ground vibration induced by blasting to the surrounding residents in advance. 

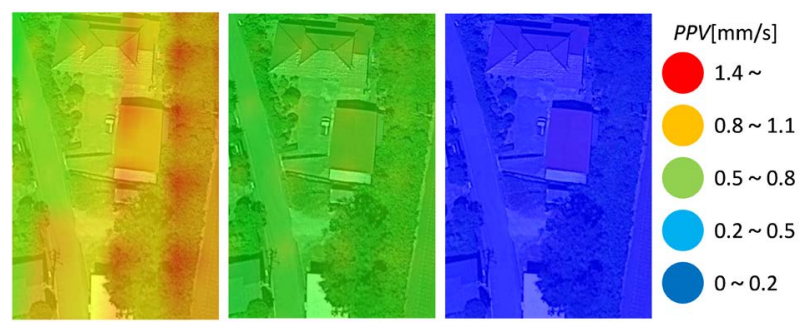

Figure 13. Enlarged view of the target residential house under each blasting condition and legend of color contours.

\section{Conclusion}

In resource extraction sites, especially urban quarries, blasting is carried out on a regular basis. Residents around urban quarry sites are routinely affected by ground vibration induced by blasting. In this study, a prediction and visualization system for ground vibration induced by blasting is developed for the purpose of reducing this adverse effect of blasting. As mentioned before, anyone with access to a browser and an internet connection can view the visualization of the predicted ground vibration. The Mikurahana quarry site and the adjacent areas are visible through the contour background so that the residents around the quarry site can know how strong ground vibration is predicted to be in their location from the contour color at a glance. Due to its implementation using Google Maps albeit with some limitations, it is possible to zoom in on the map and verify locations. This piece of information may possibly be used to alleviate worries the surrounding residents may have from the ground vibration induced by the blasting of urban quarry site. As mentioned in Chapter 4 , the employees can check the predicted vibration level and correct the blast design. With this, they can maximize the efficiency while minimizing the ground vibration on residential areas. Furthermore, it is possible that this system is announced a piece of blasting information to the surrounding residents in advance. Therefore, the possibility that the mental stress that the surrounding residents receive can be reduced is increased. It is a system that may be widely used not only for visualization of ground vibrations induced by blasting of resource extraction sites but also for research in other fields.

\section{Conflicts of Interest}

The authors declare no conflicts of interest regarding the publication of this paper.

\section{References}

[1] Klæboea, R., Amundsen, A.H., Madshus, C., Norén-Cosgriff, K.M. and Turunen-Rindel, I. (2016) Human Reaction to Vibrations from Blasting Activity-Norwegian Exposure-Effect Relationships. Applied Acoustics, 111, 49-57. https://doi.org/10.1016/j.apacoust.2016.03.021 https://www.sciencedirect.com/science/article/pii/S0003682X16300548

[2] Kumar, R., Choudhury, D. and Bhargava, K. (2016) Determination of Blast-Induced 
Ground Vibration Equations for Rocks Using Mechanical and Geological Properties. Journal of Rock Mechanics and Geotechnical Engineering, 8, 341-349. https://www.sciencedirect.com/science/article/pii/S167477551600024X

[3] Mesec, J., Kovac, I. and Soldo, B. (2010) Estimation of Particle Velocity Based on Blast Event Measurements at Different Rock Units. Soil Dynamics and Earthquake Engineering, 30, 1004-1009. https://doi.org/10.1016/j.soildyn.2010.04.011 https://www.sciencedirect.com/science/article/pii/S0267726110000904

[4] Fouladgar, N. and Hasanipanah, M. (2017) Application of Cuckoo Search Algorithm to Estimate Peak Particle Velocity in Mine Blasting. Engineering with Computers, 33, 181-189. https://link.springer.com/article/10.1007/s00366-016-0463-0

[5] Monjezi, M., Ghafurikalajahi, M. and Baharami, A. (2011) Prediction of Blast-Induced Ground Vibration Using Artificial Neural Networks. Tunnelling and Underground Space Technology, 26, 46-50.

https://doi.org/10.1016/j.tust.2010.05.002 https://www.sciencedirect.com/science/article/pii/S0886779810000799

[6] Moriyama, Y. and Kawamura, Y. (2017) Proposal of Ground Vibration Induced by Blasting Mapping Technology in Urban Quarry Site Using Web-GIS. MMIJ \& EARTH 2017, PY2-86.

[7] Moriyama, Y. and Kawamura, Y. (2018) Visualization System of Blasting Induced Ground Vibration in an Urban Quarry Using ANN and Web-GIS. MMIJ 2018, 3601-05-04.

[8] Wang, Y.Q. (2014) MeteoInfo: GIS Software for Meteorological Data Visualization and Analysis. Meteorological Applications, 21, 360-368.

https://doi.org/10.1002/met.1345

https://rmets.onlinelibrary.wiley.com/doi/full/10.1002/met.1345

[9] Hashemi, M. and Alesheikh, A.A. (2013) GIS: Agent-Based Modeling and Evaluation of an Earthquake-Stricken Area with a Case Study in Tehran, Iran. Natural Hazards, 69, 1895-1917.

https://link.springer.com/article/10.1007/s11069-013-0784-x

[10] Leh, M., Bajwa, S. and Chaubey, I. (2013) Impact of Land Use Change on Erosion risk: An Integrated Remote Sensing, Geographic Information System and Modeling. Land Degradation \& Development, 24, 409-421. https://doi.org/10.1002/ldr.1137 https://onlinelibrary.wiley.com/doi/full/10.1002/ldr.1137

[11] Gu, Q.H., Lu, C.W., Guo, J.P. and Jing, S.G. (2010) Dynamic Management System of Ore Blending in an Open Pit Mine Based on GIS/GPS/GPRS. Mining Science and Technology, 20, 132-137.

https://www.sciencedirect.com/science/article/pii/S1674526409601745

[12] Choi, Y. and Nieto, A. (2011) Optimal Haulage Routing of Off-Road Dump Trucks in Construction and Mining Sites Using Google Earth and a Modified Least-Cost Path Algorithm. Automation in Construction, 20, 982-997.

https://doi.org/10.1016/j.autcon.2011.03.015 https://www.sciencedirect.com/science/article/pii/S0926580511000422

[13] Khandelwal, M. and Singh, T.N. (2009) Prediction of Blast-Induced Ground Vibration Using Artificial Neural Network. International Journal of Rock Mechanics and Mining Sciences, 46, 1214-1222. https://doi.org/10.1016/j.ijrmms.2009.03.004 https://www.sciencedirect.com/science/article/pii/S1365160909000574

[14] Nateghi, R. (2011) Prediction of Ground Vibration Level Induced by Blasting at Different Rock Units. International Journal of Rock Mechanics and Mining Sciences, 48, 899-908. 
https://www.sciencedirect.com/science/article/pii/S1365160911000670

[15] Khandelwal, M. and Singh, T.N. (2006) Prediction of Blast Induced Ground Vibrations and Frequency in Opencast Mine: A Neural Network Approach. Journal of Sound and Vibration, 289, 711-725. https://doi.org/10.1016/j.jsv.2005.02.044

https://www.sciencedirect.com/science/article/pii/S0022460X05001823

[16] Saadat, M., Khandelwal, M. and Monjezi, M. (2014) An ANN-Based Approach to Predict Blast-Induced Ground Vibration of Gol-E-Gohar Iron Ore Mine, Iran. Journal of Rock Mechanics and Geotechnical Engineering, 6, 67-76.

https://doi.org/10.1016/j.jrmge.2013.11.001

https://www.sciencedirect.com/science/article/pii/S1674775513001157

[17] Mohamed, M.T. (2011) Performance of Fuzzy Logic and Artificial Neural Network in Prediction of Ground and Air Vibrations. International Journal of Rock Mechanics and Mining Sciences, 48, 845-851.

https://www.sciencedirect.com/science/article/pii/S1365160911000694 